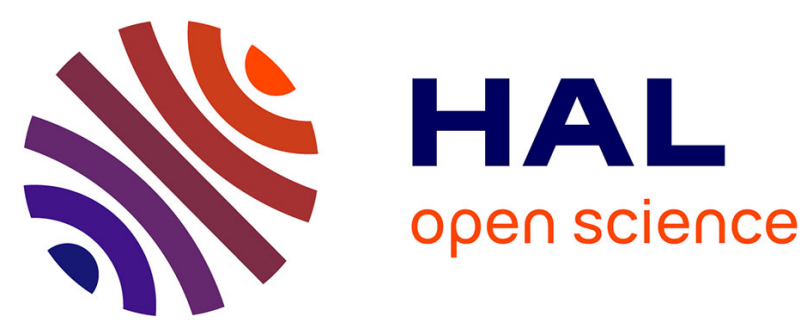

\title{
Monte Carlo simulations of prompt-gamma emission during carbon ion irradiation
}

\author{
F. Le Foulher, M. Bajard, M. Chevallier, D. Dauvergne, N. Freud, P.
} Henriquet, S. Karkar, J.M. Létang, Loïc Lestand, R. Plescak, et al.

\section{- To cite this version:}

F. Le Foulher, M. Bajard, M. Chevallier, D. Dauvergne, N. Freud, et al.. Monte Carlo simulations of prompt-gamma emission during carbon ion irradiation. IEEE Transactions on Nuclear Science, 2010, 57 (5), pp.2768-2772. in2p3-00480024

HAL Id: in2p3-00480024

https://hal.in2p3.fr/in2p3-00480024

Submitted on 3 May 2010

HAL is a multi-disciplinary open access archive for the deposit and dissemination of scientific research documents, whether they are published or not. The documents may come from teaching and research institutions in France or abroad, or from public or private research centers.
L'archive ouverte pluridisciplinaire HAL, est destinée au dépôt et à la diffusion de documents scientifiques de niveau recherche, publiés ou non, émanant des établissements d'enseignement et de recherche français ou étrangers, des laboratoires publics ou privés. 


\title{
Monte Carlo simulations of prompt-gamma emission during carbon ion irradiation
}

\author{
F. Le Foulher ${ }^{1}$, M. Bajard ${ }^{1}$, M. Chevallier ${ }^{1}$, D. Dauvergne ${ }^{1}$, N. Freud ${ }^{2}$, P. Henriquet ${ }^{1}$, S. Karkar ${ }^{3}$, J.M. Létang ${ }^{2}$, L. Lestand ${ }^{4}$, R.

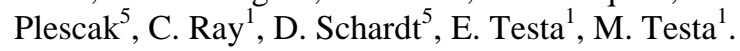

${ }^{1}$ Université de Lyon, F-69003 Lyon; Université Lyon 1 and IN2P3/CNRS, UMR 5822, Institut de Physique Nucléaire de Lyon, F-69622 Villeurbanne, France. Laboratoire de Contrôles Non Destructifs par Rayonnements ${ }^{2}$ Ionisants, INSA-Lyon, F-69621 Villeurbanne cedex, France.

${ }^{3}$ CPPM, Aix-Marseille Université, CNRS/IN2P3, Marseille, France.

${ }^{4}$ LPC Clermont, Université Blaise Pascal, CNRS/IN2P3, F-63177 Aubiere, France.

${ }^{5}$ Gesellschaft für Schwerionenforschung (GSI), D-64291 Darmstadt, Germany

Corresponding author to provide e-mail: f.le-foulher@ipnl.in2p3.fr

\begin{abstract}
Monte Carlo simulations based on the Geant4 toolkit (version 9.1) were performed to study the emission of secondary prompt-gamma rays produced by nuclear reactions during carbon ion-beam therapy. These simulations were performed along with an experimental program and instrumentation developments which aim at designing a prompt-gamma ray device for realtime control of hadrontherapy. The objective of the present study is twofold: firstly, to present the features of the prompt-gamma radiation in the case of carbon ion irradiation; secondly, to simulate the experimental setup and to compare measured and simulated counting rates corresponding to four different experiments. For each experiment, we found that simulations overestimate prompt-gamma ray detection yields by a factor of 12 . Uncertainties in fragmentation cross sections and binary cascade model cannot explain such discrepancies. The so-called "photon evaporation" model is therefore questionable and its modification is currently in progress.
\end{abstract}

\section{I.INTRODUCTION}

Hadrontherapy consists in treating cancerous tumors with proton or light ion beams. This technique is very advantageous for the treatment of deep-seated tumors thanks to a ballistic precision much higher than in conventional radiotherapy with X-rays. In addition, the use of light ions (such as carbon ions) presents optimum biological effectiveness in the target volume, allowing the treatment of radioresistant cancers. Clinical experience in carbon ion therapy has been accumulated for more than ten years in Japan and Germany with extremely positive results and hadrontherapy developments are presently planned worldwide [1,2].

To make the best use of the hadrontherapy advantages, real-time control of the dose delivered to the patient is highly desirable. Since no primary radiation emerges from the patient, in vivo imaging must be performed exploiting secondary radiations produced during nuclear reactions. Two kinds of radiations can be identified: i) prompt radiation (emitted during nuclear reactions at a time scale much smaller than one picosecond) dominated by photon, proton and neutron emissions and ii) the radioactivity of some fragments, in particular $\beta+$ emitters.

For both proton and carbon ion beams, it has been demonstrated that the $\beta+$ activity distribution can be visualized at the end of the irradiation by means of positron emission tomography (PET) to provide valuable information for quality assurance $[3,4,5]$. In-beam PET for hadron therapy has been successfully used at GSI until 2008 to control the dose after irradiations. However, by using the actual system, it seems very difficult to foresee a real-time control with this technique, mainly because of the very low $\beta+$ activity induced during the irradiation and the intrinsic problem of the nuclear lifetimes, during which positron emitters undergo a metabolic washout.

Prompt-gamma ray imaging appears as a good candidate to provide real-time information about the local dose both for proton $[6,7]$ and carbon ion [8] therapy. Indeed the prompt-gamma emission point distribution is tightly correlated to the dose distribution and the emission yields are of the same order of magnitude as the number of fragmentation events, which potentially constitutes a sufficiently intense source for in-beam single photon emission tomography.

The present paper aims at presenting the comparison between our experimental data with Monte Carlo simulation results obtained with the Geant4 toolkit (version 9.1) [9]. The second section is dedicated to a brief presentation of the nuclear physics involved during ion beam irradiations at hadrontherapy energies. Our experimental setup is also described with a highlight on the 
need of Time-Of-Flight to discriminate gamma rays from background. The comparison between experimental and simulated results and the need for Geant4 hadronic models are discussed in the third section.

\section{II.PHYSICS CASE}

\section{A.Physics of promp-gamma rays}

Depending on their energy, prompt-gamma rays are emitted during the successive stages of nuclear fragmentation $[10,11,12]$. As a rough description, the continuum energy spectrum can be divided into three components. High energy gamma-rays (E > 30 $\mathrm{MeV}$ ) are mainly produced during nucleon-nucleon bremsstrahlung at the very beginning of the reaction. Between 10 and 30 $\mathrm{MeV}$, prompt-gamma rays are emitted during the decay of giant resonances, mostly Giant Dipole Resonances (GDR), a collective oscillation between protons and neutrons in the nucleus. Below $10 \mathrm{MeV}$, the main contribution for gamma production is statistical photons coming from the remaining excited fragments at the final stage of the reaction. Lastly, at the end of the evaporation cascade, photons with discrete energies are emitted. The prompt-gamma ray spectrum is dominated by statistical photons whose energy ranges from a few $\mathrm{keV}$ up to $10-15 \mathrm{MeV}$.

\section{B.Geant4 physics list}

The whole nuclear collision process can be described in four steps: the nuclear collision cross section, the intranuclear interaction, the pre-equilibrium stage and the deexcitation stage. Several models are available in Geant 4 for the two first steps: concerning the nuclear collision cross sections, the Shen formula was used in this work. The binary cascade model was used to describe the inelastic interaction between protons, neutrons and light ions with target nuclei. In this model a three-dimensional intranuclear cascade (INC) propagates particles within the nucleus, which accounts for the energy transfer to nuclear matter.

The INC is used for projectile larger than $50 \mathrm{MeV} / \mathrm{u}$ leading to the formation of excited fragments, later managed by the precompound model. At lower energy, the INC is not valid anymore and a simple fusion of the projectile and the target nuclei is applied leading to the formation of a compound nucleus which will be managed by the precompound model. The precompound model is used as a smooth transition from highly excited nuclear matter to statistical equilibrium (exciton model). This model leads to the emission of light particles ( $\mathrm{p}, \mathrm{n}, \mathrm{d}, \mathrm{t},{ }^{3} \mathrm{He}$ and $\alpha$ )

When this statistical equilibrium is reached two decay modes are taken into account by default in Geant4: the evaporation decay (emission of light particles and gamma rays) based on the Weisskopf-Ewing model and the photon evaporation channel at the end of the de-excitation cascade. Two other decay models can be called to treat relatively high excitation energy (above 3 $\mathrm{MeV} / \mathrm{u})$ : the statistical multi-fragmentation for heavy nuclei $(\mathrm{A}>17)$ and the Fermi break-up for light nuclei $(\mathrm{A}<17)$.

If the fragment is still excited at the end of the evaporation cascade, an additional gamma is emitted in order to complete the de-excitation. The prompt-gamma energy spectrum predicted by Geant 4 is dominated by a broad component ranging from a few $\mathrm{keV}$ up to $10-20 \mathrm{MeV}$, in which some discrete transitions appear, in particular carbon transitions. Nevertheless the number of hard photons is far from being negligible [13]. As regards the electromagnetic processes, the standard electromagnetic physics package was selected in our work including standard electromagnetic process (Compton scattering, pair production and photoelectric effect for photons, multiple scattering and ionization for charged particles and electron and positron bremsstrahlung).

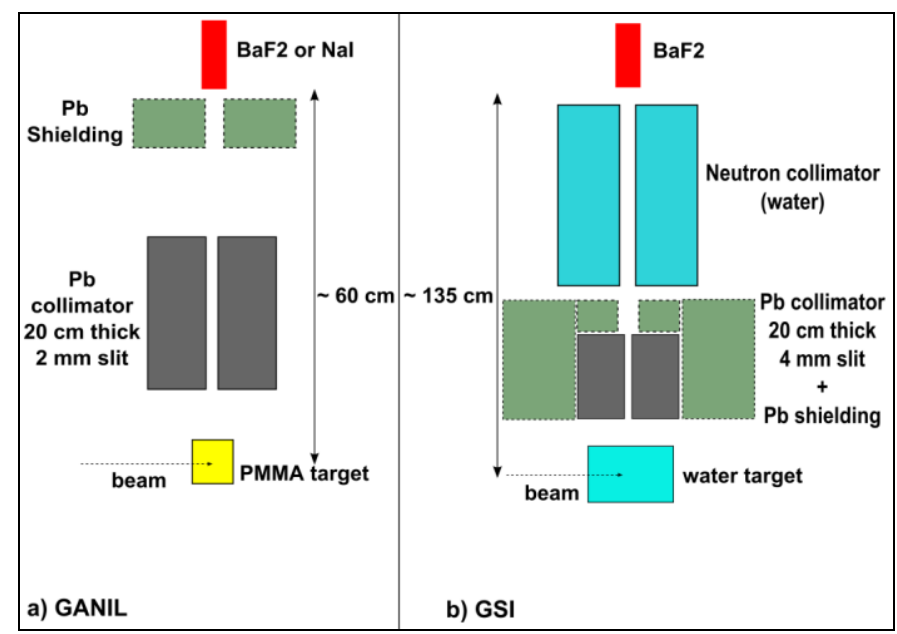

Fig. 1 Set-up reproduced in Geant4 simulations: a) Diagram of the experimental set-up used at GANIL. b) Diagram of the experimental set-up used at GSI. 


\section{B.Experimental set-up}

In order to detect prompt-gamma rays, four experiments were carried out: two at the GANIL facility with relatively low energy beams $\left(73 \mathrm{MeV} / \mathrm{u}{ }^{13} \mathrm{C}^{6+}\right.$ and $95 \mathrm{MeV} / \mathrm{u}{ }^{12} \mathrm{C}^{6+}$ ) and two at GSI with higher energy beams (300 MeV/u and $310 \mathrm{MeV} / \mathrm{u}$ ${ }^{12} \mathrm{C}^{6+}$ ). The experimental set-up was reproduced in our simulations for the four experiments (see figure 1).

This set-up consists in a collimated detection system placed at $90^{\circ}$ from the beam line to determine the emission point of the prompt-gamma rays, as a function of the beam penetration depth in the target $[8,13]$. In the case of the GANIL experiments, the ion beam irradiated a $5 \mathrm{~cm} \times 5 \mathrm{~cm} \times 5 \mathrm{~cm}$ PMMA target. A $5 \mathrm{~cm}$ in diameter, $5 \mathrm{~cm}$ thick $\mathrm{NaI}(\mathrm{Tl})$ detector was used in a first experiment, and then replaced by a $9 \mathrm{~cm}$ in diameter, $16 \mathrm{~cm}$ thick $\mathrm{BaF}_{2}$ scintillator. The additional lead shielding was used in order to reduce the background signal coming from gamma rays scattered by surrounding materials. In the GSI experiments the ion beam was impinging on a $12 \mathrm{~cm}$ wide, $20 \mathrm{~cm}$ high and $21 \mathrm{~cm}$ thick water target. The $\mathrm{BaF}_{2}$ detector was placed $135 \mathrm{~cm}$ away from the beam line behind a $20 \mathrm{~cm}$ thick lead collimator. Additional lead shielding was added around the collimator. The experimental room was not taken into account in the simulations presented in this paper.

\section{C.Detection technique}

Many neutrons are produced during the collisions between the primary or secondary particles and the target nuclei. A key point for the detection of prompt-gamma rays is the ability to discriminate prompt-gamma signals from neutron signals in the detector. The Time-Of-Flight (TOF) technique allowed this identification in our experiments [8]. In all experiments the start signal is provided by the detection of a particle by the scintillator. The stop signal used depends on the structure of the beam: (i) at GANIL where the beam is pulsed, the stop signal was provided by the cyclotron high-frequency (HF) (beam pulse of $\sim 1 \mathrm{~ns}$ every $80 \mathrm{~ns}$ ); (ii) at SIS-GSI synchrotron where a continuum extraction mode was used (beam pulse of $8 \mathrm{~s}$ every $10 \mathrm{~s}$ ), the beam was kept at low intensity $\left(\sim 10^{5} \mathrm{pps}\right)$ to allow the use of two thin plastic scintillators placed next to the exit window of the beam, to provide the stop signal.

The choice of setting the lowest count-rate detector giving the start signal was adopted to minimize the number of void events for which a start signal does not have a corresponding stop signal. This choice requires the stop to be suitably delayed.

Moreover, as low-energy deposition in the detector is likely to be dominated by neutrons and scattered gamma-rays, the TOF technique is coupled with an energy selection above typically 1 or $2 \mathrm{MeV}$ photon equivalent energy. Figure 2 shows a time $v s$. energy spectrum measured with a $\mathrm{BaF}_{2}$ scintillator with a $95 \mathrm{MeV} / \mathrm{u}^{12} \mathrm{C}^{6+}$ beam. The origin of time corresponds to the entrance of the beam in the target. Energy and time selections shown by the red rectangle allow the selection of prompt-gamma rays, which represent a very minor part of the detected signals. It must be noticed that the neutron and scattered gamma-ray background can be reduced but not suppressed by the energy selection, and that the measurement of a gamma-ray yield requires the background suppression by means of a measurement upstream the target.

In the simulations, the start signal is provided by the impact time of the projectile on the target and the stop signal is triggered by the detection of particles in the photon detector.

\section{III.COMPARISON BETWEEN SIMULATION AND MEASUREMENTS}

\section{A. Counting rates}

Comparisons between simulations and measurements were carried out for each experiment. In all cases, a large discrepancy appears between the measured and simulated counting rates.

This is clearly illustrated by Figure 3, which presents measured and simulated spectra of the energy deposited in a $\mathrm{NaI}(\mathrm{Tl})$ detector. The measured spectrum corresponds to a Time-of-Flight window of prompt-gamma rays that is similar to the one presented on Figure 2. Geant4 simulations overestimate the detection of prompt-gamma rays by a nearly constant factor of 12 over the whole energy spectrum beyond $1 \mathrm{MeV}$. Note that the simulated spectrum does not take into account the energy resolution of the detector, which explains that some discrete lines appear on this spectrum whereas only the $511 \mathrm{keV}$ positron annihilation peak can be observed on the measured one. The small bump which lies between 0 and $500 \mathrm{keV}$ in the measured spectrum is likely to correspond to neutrons scattered by the environment (table, walls, floor, ceiling), which was not taken into account in the simulations. 


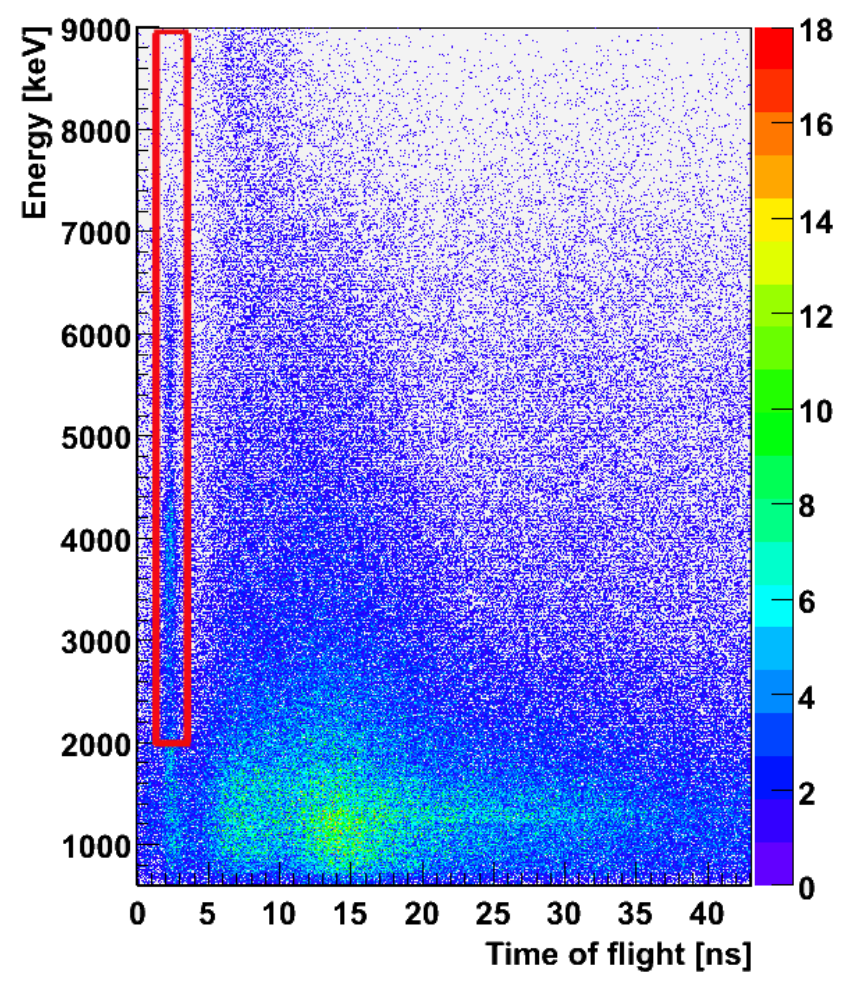

Fig. 2 Time-energy spectrum measured with a $\mathrm{BaF}_{2}$ scintillator at the GANIL facility with a $95 \mathrm{MeV} / \mathrm{u}{ }^{12} \mathrm{C}^{6+}$ ion beam. The set-up used is represented in figure 1, origin of time corresponds to the entrance of the beam in the target.

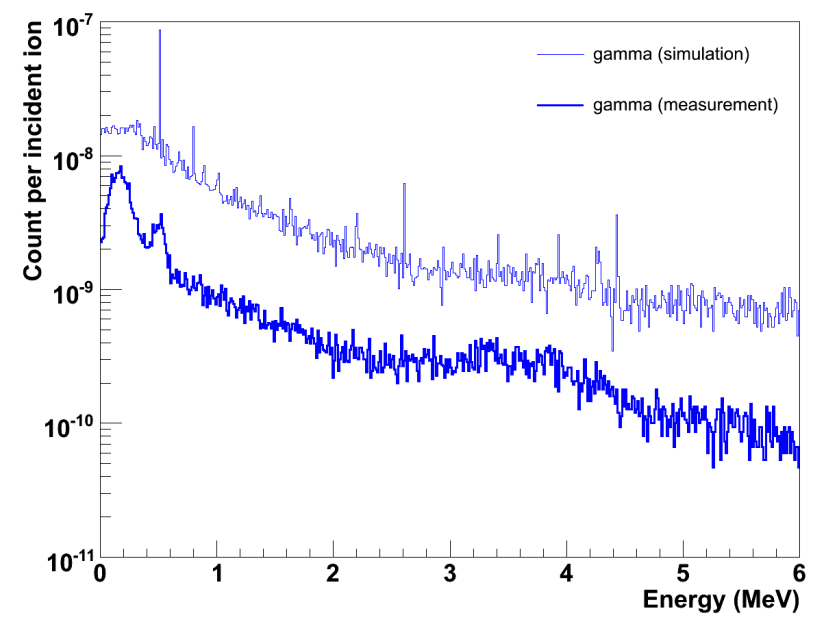

Fig. 3 Measured and simulated spectra of the energy deposited by prompt-gamma rays in a $\mathrm{NaI}(\mathrm{Tl})$ detector $\left({ }^{13} \mathrm{C}\right.$ ion beam at $73 \mathrm{MeV} / \mathrm{u}$, PMMA target).

\section{B. Promp-gamma ray profile}

In order to obtain the longitudinal profile of prompt-gamma rays, measurements and simulations were performed for several depths in the target (corresponding to various positions of the collimator with respect to the target). The results for two experiments are presented in this paper: the first one with a low-energy beam $\left(95 \mathrm{MeV} / \mathrm{u}^{12} \mathrm{C}^{6+}\right)$ at GANIL and the second at the GSI facility with a high-energy beam $\left(310 \mathrm{MeV} / \mathrm{u}^{12} \mathrm{C}^{6+}\right)$. The measured and simulated gamma profiles are presented respectively at the top and at the middle of the Figure 4 (the error bars correspond to statistical fluctuations). The bottom spectra show the emission yield and the longitudinal profile of the fragmentation events as a function of depth in the target (along with the dose deposition profiles).

For each position, measurements are obtained by integrating the counts in the photon detector according to the time and energy selections. Since the penetration depth of a ${ }^{12} \mathrm{C}^{6+}$ beam is $20 \mathrm{~mm}$ in PMMA at $95 \mathrm{MeV} / \mathrm{u}$ and $18 \mathrm{~cm}$ in water at 310 $\mathrm{MeV} / \mathrm{u}$, a strong correlation is observed in both cases between the gamma profile and the ion range. Moreover, the Bragg peak 
position coincides with a gamma emission enhancement, which is an important feature for the spatial resolution of our technique. Note however that for the $310 \mathrm{MeV} / \mathrm{u}$ case, the poorer correlation is mainly due to the slit width $(4 \mathrm{~mm})$ and the target-todetector distance $(135 \mathrm{~cm})$ which were larger compared to the $95 \mathrm{MeV} / \mathrm{u}$ case.

For each experiment, the shape of the profile is not perfectly well reproduced. At the low energy experiment, the measured counting rates increase with the ion penetration depth, while the simulated counting rates decrease. At the high energy experiment, the measured gamma profile is more flat, but, although the statistics are poor, the counting rates seem to increase also near the Bragg peak. If one takes into account the statistical fluctuations, the simulated profile can be considered as flat all along the ion range. Thus, simulations fail to reproduce the Bragg peak enhancement in the prompt-gamma ray profile.

At the bottom of Figure 4, the simulated distributions of fragmentation points present a tail behind the Bragg peak. Indeed, secondary particles, mostly neutrons, induce secondary fragmentation beyond the Bragg peak location. These secondary fragmentations have an effect on the contrast between counting rates measured inside and outside the primary ion beam path. This effect is more important at high energy and provides a physical limitation of the contrast. However in the GSI experiment, measurements after the Bragg peak location were obtained outside the target thus this effect can not be observed. At lower energy, the number of nuclear fragmentations is smaller beyond the Bragg peak, which explains why the contrast measured at GANIL is higher. In the calculated spectrum to reproduce the GANIL experiment (bottom left), a discontinuity is clearly visible at $50 \mathrm{MeV} / \mathrm{u}(5 \mathrm{~mm}$ residual range) for particle emission. Gamma production slightly decreases while neutron emission increases. This break can be explained by a model change for particles emission rather than a real physical behaviour. It is responsible for the simulated counting rate decrease as a function of ion penetration depth.

$95 \mathrm{MeV} / \mathrm{u}^{12} \mathrm{C}$-ions in PMMA (GANIL)

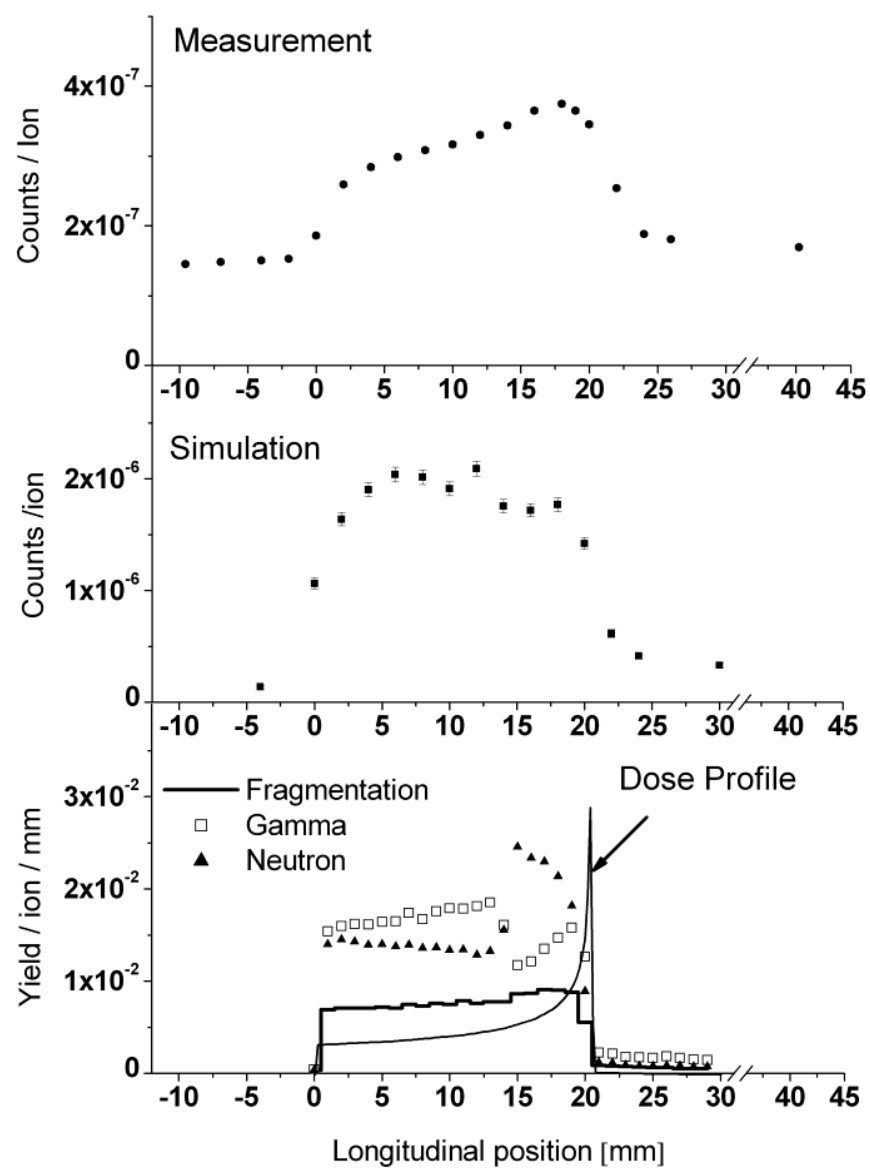

$310 \mathrm{MeV} / \mathrm{u}^{12} \mathrm{C}$-ions in water (GSI)

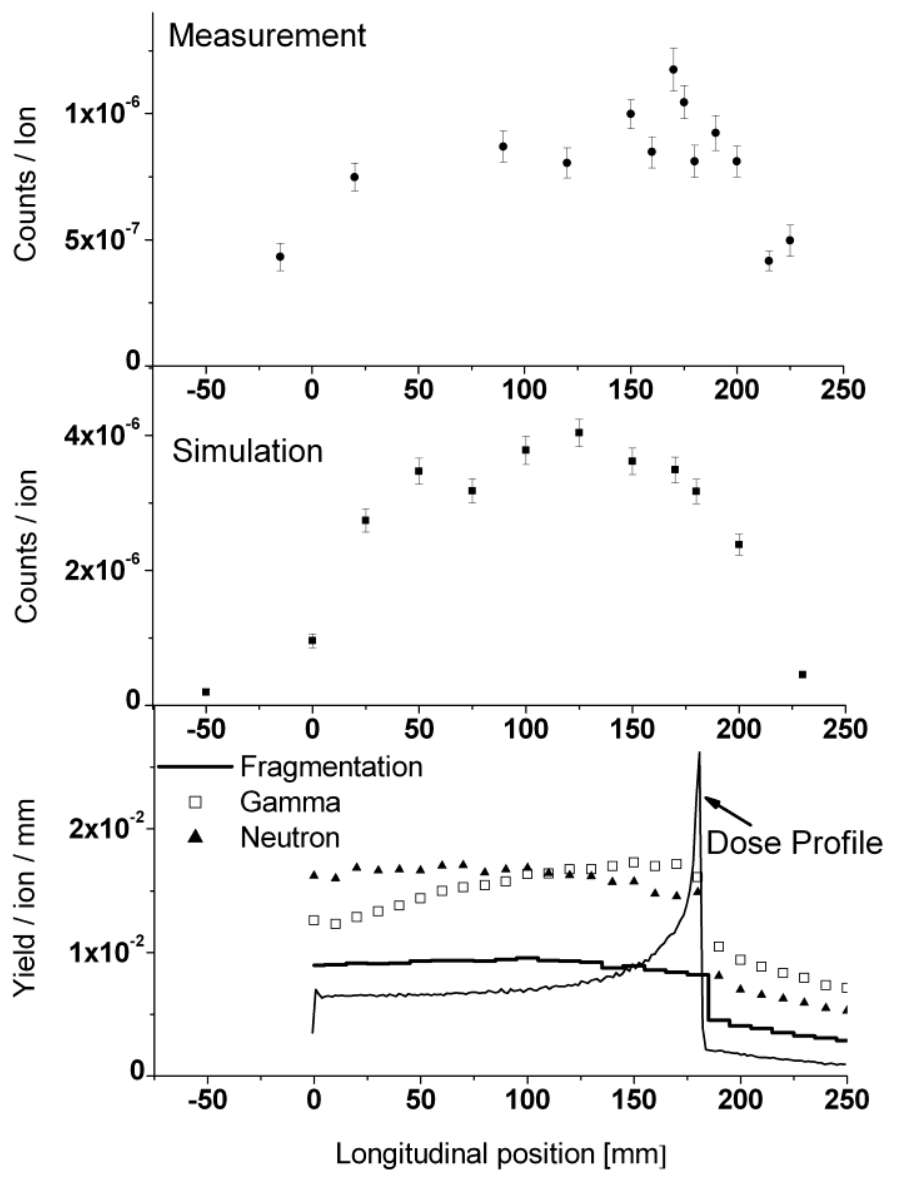

Fig. 4 Depth profiles Spectra obtained in two experimental conditions, ${ }^{12} \mathrm{C}$ at $95 \mathrm{MeV} / \mathrm{u}$ and $2 \mathrm{~mm}$ slit (left), and ${ }^{12} \mathrm{C}$ at $310 \mathrm{MeV} / \mathrm{u}$ and $4 \mathrm{~mm}$ slit (right). Comparison between the measured gamma-ray profiles at GANIL and GSI (top) and simulated with GEANT4 (middle). The bottom curves show the emission yield and the longitudinal profile of the fragmentation events as a function of depth in the target. The dose deposition profile is superimposed (arbitrary units) 


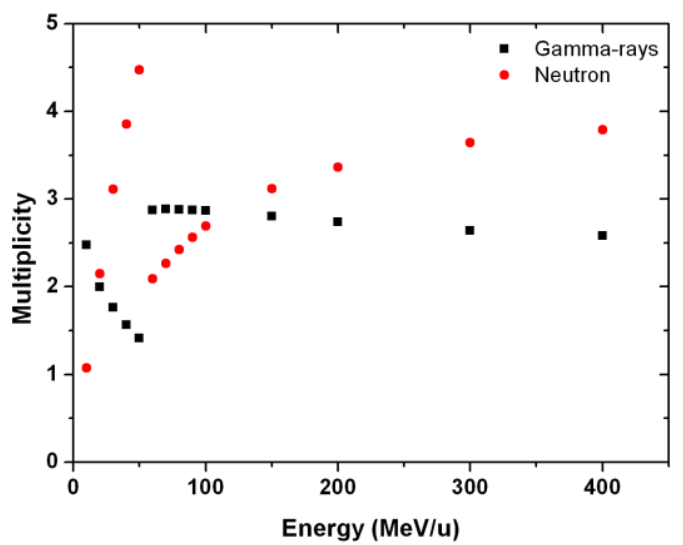

Fig. 5. Multiplicity of gamma-rays (black) and neutron (red) for the inelastic reaction ${ }^{12} \mathrm{C} \rightarrow{ }^{12} \mathrm{C}$ as a function of the incident energy.

\section{IV.DISCUSSION}

The discrepancy observed between simulations and measurements may originate from (i) the fragmentation cross sections and/or (ii) the gamma emission multiplicity per inelastic nuclear process. The validity of these data and the corresponding process implementation in Geant 4 have therefore to be examined. The cross sections used in Geant 4 have been inferred by simulating the irradiation of a thin target with mono-energetic ion beams from $10 \mathrm{MeV} / \mathrm{u}$ up to $400 \mathrm{MeV} / \mathrm{u}$. The nuclear fragmentation cross sections extracted from these simulations were then compared with experimental data [14]. A satisfactory agreement - to within 10 percent - was found. Thus we conclude that the cross sections used in Geant 4 cannot explain the discrepancy in the counting rates of the prompt-gamma rays. This discrepancy must be due to the gamma multiplicity per nuclear fragmentation.

Moreover, the sharp discontinuity of the production of gamma-rays and neutrons seen in Figure 4 (bottom left) originates from a sudden change of the gamma-ray and neutron multiplicities per nuclear fragmentation occurring for $50 \mathrm{MeV} / \mathrm{u}{ }^{12} \mathrm{C}$ incident energy, as shown in Figure 5. Below this energy, the gamma-ray yield steeply decreases when the energy increases, whereas the neutron yield increases. From $50 \mathrm{MeV} / \mathrm{u}$ up to $400 \mathrm{MeV} / \mathrm{u}$, the yield of prompt-gamma rays decreases slightly but the discontinuity at $50 \mathrm{MeV} / \mathrm{u}$ is not physically realistic and comes from a change of model already mentioned in section II.B. It may explain why the gamma yield maximum detected at the end of the ion path is not reproduced in the simulations.

\section{V.CONCLUSION}

With the physics list used in this work, Geant4 (version 9.1) is able to reproduce the fragmentation yields of carbon ions at energies of relevance for hadrontherapy. However, the gamma evaporation models overestimate the total emission yield by a factor of 12, which was reproducible in several experiments. Moreover, the discontinuity in the gamma and light particle yields at $50 \mathrm{MeV} / \mathrm{u}{ }^{12} \mathrm{C}$ beam energy confirms that the change of nuclear collision model from INC (at high energy) to a simple fusion of projectile and target nuclei is not satisfactory. Further efforts are undertaken to improve these models and recent simulations using the QMD (Quantum Molecular Dynamics) model instead of the binary cascade model, for projectiles heavier than protons and neutrons, seem to succeed in reproducing the gamma emission enhancement in the prompt-gamma ray profile. However, counting rates of prompt-gamma rays stay unchanged, because de-excitation models are the same as in the case of the binary cascade model. Comparisons of the Geant4 toolkit with other existing codes such as MCNPX, PHITS or FLUKA are planned. This is a key issue for the development of an online gamma-ray based monitoring system for ion therapy.

These studies are supported by the French GDR MI2B, the Rhône-Alpes Regional Research Program for Hadrontherapy and the French ANR SimCa2.

\section{REFERENCES}

[1] U. Amaldi and G. Kraft, "Radiotherapy with beams of carbon ions", Rep. Prog. Phys., vol. 68, pp. 1861-1882, July 2005.

[2] G. Kraft, "Ion beam therapy in Europe", APPLICATION OF ACCELERATORS IN RESEARCH AND INDUSTRY: Twentieth International Conference. AIP Conference Proceedings, vol. 1099, pp. 429-434, 2009. 
[3] W. Enghardt et al., "Charged hadron tumour therapy monitoring by means of PET", Nucl. Instrum. Methods Phys. Res. A, vol. 525, pp. 284-288, June 2004.

[4] K. Parodi et al., "PET imaging for treatment verification of ion therapy: Implementation and experience at GSI Darmstadt and MGH Boston", Nuclear Instruments and Method A, vol. 591, pp. 282-286, June 2008.

[5] T. Nishio et al., "Experimental verification of proton beam monitoring in a human body by use of activity image of positron-emitting nuclei generated by nuclear fragmentation reaction", Radiol. Phys. Technol., vol. 1, pp. 44-54, Jan 2008.

[6] C. H. Min, C. H. Kim, M. Y. Youn, J. W. Kim, "Prompt gamma measurements for locating the dose falloff region in the proton therapy", Appl. Phys. Lett. vol. $89,183517,2006$.

[7] J. C. Polff et al., "Measurement and calculation of characteristic prompt gamma ray spectra during proton irradiation", Phys. Med. Biol. Vol 54, pp N519527, Oct. 2009.

[8] E. Testa et al., "Monitoring the Bragg peak location of $73 \mathrm{MeV} / \mathrm{u}$ carbon ions by means of prompt gamma-ray measurements", Applied Physics Letters, vol. 93, 093506, Sep. 2008.

[9] S. Agostinelli at al., "Geant4 - a simulation toolkit", Nucl. Instrum. Methods Phys. Res. A, vol. 506, pp. 250-303, Jul. 2003.

[10] H. Nifenecker et al., High energy gamma-ray emission in heavy-ion reactions, Progress in Particle and Nuclear Physics, vol. 23, pp. 271-355, 1989.

[11] B. L. Berman and S. C. Fultz, "Measurements of the giant dipole resonance with monoenergetic photons", Rev. Mod. Phys., vol. 47, pp. 713-761, July 1975.

[12] J. F. Mollenauer, "Gamma-Ray Emission from Compound Nucleus Reactions of Helium and Carbon Ions”, Phys. Rev., vol. 127, pp 867-879 Aug. 1962.

[13] E. Testa et al., "Dose profile monitoring with carbon ions by means of prompt-gamma measurements", Nuclear Instruments and Method, vol. 267, pp. 993996, Mar. 2009.

[14] W. Shen et al., "Total reaction cross section for heavy-ion collisions and its relation to the neutron excess degree of freedom", Nuclear Physics, Section A, vol. 491, pp. 130-146, Jan. 1989. 